\title{
Orofacial Crohn's Disease: A Case Report
}

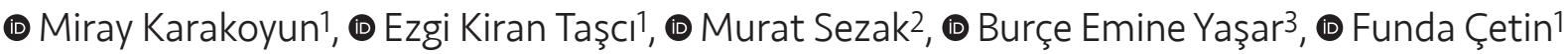

${ }^{1}$ Ege University Faculty of Medicine, Department of Pediatric Gastroenterology, Division of Hepatology and Nutrition, İzmir, Turkey

2Ege University Faculty of Medicine, Department of Pathology, İzmir, Turkey

${ }^{3}$ Ege University Faculty of Medicine, Department of Pediatrics, İzmir, Turkey

\begin{abstract}
Crohn's disease (CD) is a chronic disease of the digestive system. It is characterized by lesions predominantly located in the small intestine and colon, although they may also occur in any segment of the gut, including the oral cavity. The involvement of oral mucosa in CD may be underreported, as up to $42 \%$ of pediatric patients with CD were found to have oral lesions after undergoing a thorough oral examination. Here, we present a case of $C D$ in which the patient was referred to a dentist due to non-healing aphthous ulcers in the mouth. Our patient, a 16-year-old boy, was admitted to the dentistry clinic with swelling of the oral mucosa and the lips which had been ongoing for 3 months. The patient was referred to our department due to the non-response of the mucosal lesions to repeated cycles of medical treatment. Colonoscopy revealed a cobblestone appearance especially in the left colon, partly normal mucosa, and exudative ulcers. Biopsy samples showed increased inflammatory cell infiltration in the lamina propria and cryptitis in some of the crypts. A close collaboration between gastroenterologists and dentists is useful when addressing the diagnosis and appropriate management of these patients.
\end{abstract}

Keywords: Crohn's Disease, oral cavity, dentist

\section{Introduction}

Crohn's disease (CD) is a chronic disease of the digestive system. It is characterized by lesions predominantly located in the small intestine and colon, although they may also occur in any segment of the gut, including the oral cavity (1). Due to the prolonged course of the disease, diagnosis may be problematic; however, the findings of lesions in the oral mucosa may help to raise suspicion. The clinical spectrum of orofacial CD includes hyperplasia, cobble-stoning, ulceration of the buccal and gingival mucosa and swelling of the lips and face. The involvement of oral mucosa in CD may be underreported, as up to $42 \%$ of pediatric patients with $C D$ were found to have oral lesions after undergoing a thorough oral examination (2). Here, we present a case of $C D$ in which the patient was referred to a dentist due to non-healing aphthous ulcers in the mouth.

\section{Case Report}

Our patient, a 16-year-old boy, was admitted to the dentistry clinic with swelling of the oral mucosa and the lips which had been ongoing for 3 months. The patient was referred to our department due to the non-response of the mucosal lesions to repeated cycles of medical treatment. We were informed of the patient's history of anal fissure and diarrhea complaints which had occurred 1 year previously. There were no features in the medical history of the patient and his parents, and the patient had 2 healthy siblings. In physical examination, his weight was $55 \mathrm{~kg}$ (25-50 p), height $174 \mathrm{~cm}$ (50-75 p), cardiac pulse 96/minimum, and arterial blood pressure $120 / 80 \mathrm{mmHg}$. We observed a cobblestone appearance inside the mouth and swelling in the lips. His anal examination revealed two fissures. In the examinations for definitive diagnosis of inflammatory bowel diseases (IBD), 
sedimentation was found to be $60 \mathrm{~mm} / \mathrm{h}$, and C-reactive protein $3.5 \mathrm{mg} / \mathrm{L}$. The patient's liver and kidney function tests were normal. Immunoglobulin G, A and M levels were 1.260 $\mathrm{mg} / \mathrm{dL}, 390 \mathrm{mg} / \mathrm{dL}$ and $66.9 \mathrm{mg} / \mathrm{dL}$, respectively. Whole blood count analyses were Hemoglobin: $10.4 \mathrm{gr} / \mathrm{dL}$, hematocrit: 32.2\%, Platelets: 304.000 , Fe: 40 mg/dL and ferritin: 49 ng/ $\mathrm{mL}$. Upper and lower gastrointestinal system endoscopies were performed on the patient due to suspected IBD. Colonoscopy revealed a cobblestone appearance especially in the left colon, partly normal mucosa, and exudative ulcers. Biopsy samples showed increased inflammatory cell infiltration in the lamina propria and cryptitis in some of the crypts (Figure 1). The offer of Buccal biopsy was not consented by the teenage boy. Directed by the patient's medical history, physical examination, laboratory and biopsy findings, the patient was diagnosed with CD. The disease was extensive with PCDAI score of 30 and methylprednisolone treatment (60 mg/day) and mesalazine (40 mg/ $\mathrm{kg} / \mathrm{d}$ ) were initiated. During an observation period of two weeks, the acute phase reactants of the patient normalized completely, the cobblestone appearance was restored and swelling receded. The dosage of methylprednisolone was decreased by $5 \mathrm{mg}$ per week for four weeks, and $2 \mathrm{mg} / \mathrm{kg} /$ day of azathioprine was added to the treatment. The patient has been followed up with azathioprine maintenance and has been in clinical and laboratory remission for the last 18 months. Informed consent was obtained from the family.

\section{Discussion}

We report a case of a patient with CD presenting with cobblestone-like oral lesions. We confirmed the diagnosis of

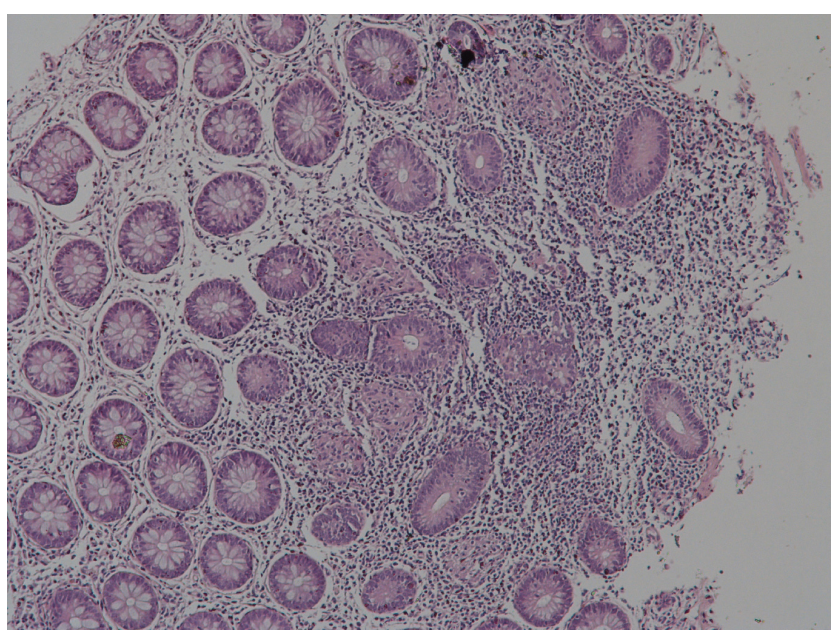

Figure 1. Histopathological examination (colon) Explanation: Hematoxylin-eosin staining x10 magnification. In lamina propria; non-caseating microgranulomas, increased infiltration of focal lymphocytes and cryptitis (focal active colitis)
CD by performing a colonoscopy. Oral lesions immediately responded to high dose steroid treatment.

A wide variety of disease-specific oral lesions have been described in patients with intestinal CD. These include swelling of the lips and buccal mucosa, cobble-stoning, mucogingivitis, deep linear ulceration or mucosal tags (3).

The prevalence of oral manifestations in patients with CD varies between $0 \%$ and $9 \%$ (4) in adults; however, it is more prevalent in children. In a prospective study of 48 children presenting with $C D$, clinicians accurately identified the presence of oral CD in less than $50 \%$ of children with disease-specific lesions.

Studies with larger sample sizes have also suggested that the finding of certain oral lesions may be indicative of $C D$, especially in children. In a prospective study conducted in Brazil comparing $62 \mathrm{CD}$ patients with a control group, oral lesions were found in $14.5 \%$ of those patients with CD and in $9.6 \%$ of the control group, showing a low prevalence and an insignificant difference between the patients and controls (5). Bezerra et al. (6), assessing the oral mucosa of 100 adult patients with CD and ulcerative colitis for a period of 5 years, observed orofacial findings in only 7 patients, concluding that the association between CD and orofacial findings is not as strong as has been reported (6).

Some oral lesions have been postulated as possible indicators of the presence of CD. Rehberger et al. (4) described the case of a 20-year-old patient with painful intra-oral lesions. On endoscopy, extensive lesions of the gastrointestinal tract were seen, and biopsies confirmed the diagnosis of CD. In our case, we suspected CD due to nonhealing painful oral lesions and confirmed the diagnosis by colonoscopic biopsies.

There is no evidence-based algorithm for the treatment of orofacial CD. Elemental diets appear to have variable outcomes. One case of facial and ileocolic CD showed improvement of the disease with total nutrition (7). Several case reports document remission of oral symptoms with topical or systemic steroids used in conjunction with aminosalicylic acid or mercaptopurine $(8,9)$. There have also been several cases of orofacial CD refractory to steroids which were treated with infliximab (10). Since our patient had extensive disease, systemic corticosteroids were applied as a first line treatment according to the ECCO guidelines (11).

Many patients, particularly children, have involvement of the mouth when presenting with CD. Although usually subclinical, self-resolving and not requiring specific treatment, these disease-specific manifestations commonly harbor diagnostic material. Expert evaluation of the oral cavity is a 
useful adjunct in patients presenting with suspected IBD. A close collaboration between gastroenterologists and dentists is useful when addressing the diagnosis and appropriate management of these patients.

\section{Ethics}

Informed Consent: Informed consent was obtained from the family.

Peer-review: Externally peer-reviewed.

\section{Authorship Contributions}

Surgical and Medical Practices: M.K., E.K.T., M.S., B.E.Y., F.Ç., Concept: M.K., E.K.T., M.S., B.E.Y., F.Ç., Design: M.K., E.K.T., M.S., B.E.Y., F.Ç., Data Collection or Processing: M.K., E.K.T., M.S., B.E.Y., F.Ç., Analysis or Interpretation: M.K., E.K.T., M.S., B.E.Y., F.Ç., Literature Search: M.K., E.K.T., M.S., B.E.Y., F.Ç., Writing: M.K., E.K.T., M.S., B.E.Y., F.Ç.

Conflict of Interest: No conflict of interest was declared by the authors.

Financial Disclosure: The authors declared that this study received no financial support.

\section{References}

1. Fatahzadeh M, Schwartz R, Kapila R, Rochford C. Orofacial Crohn's Disease: An oral enigma. Acta Dermatovenerol Croat 2009;17:289-300.
2. Harty $S$, Fleming $P$, Rowland $M$, et al. A prospective study of the oral manifestations of Crohn's disease. Clin Gastroenterol Hepatol 2005;3:886-91.

3. Rowland $M$, Fleming $P$, Bourke B. Looking in the mouth for Crohn's Disease. Inflammatory Bowel Dis 2010;16:332-7.

4. Rehberger A, Püspök A, Stallmeister T, Jurecka W, Wolf $K$. Crohn's disease masquerading as aphthous ulcers. Eur Dermatol 1998;8:274-6.

5. Pittock S, Drumm B, Fleming P, et al. The oral cavity in Crohn's disease. J Pediatr 2001;138:767-71.

6. Bezerra R, Cruz C, Catapani WR. Evaluation of the oral mucosa of Crohn's disease outpatients: A case control study. Gastroenterol Hepatology Open Access 2014;1:00013.

7. Lim S, Dohil R, Meadows N, et al. Treatment of orofacial and ileocolonic Crohn's disease with total enteral nutrition. J R Soc Med 1998;91:489-90.

8. Galbraith SS, Drolet BA, Kugathasan S, et al. Asymptomatic inflammatory bowel disease presenting with mucocutaneus findings. Pediatrics 2005;116:439-44.

9. Ganesh R, Suresh N, Ezhilarasi S, et al. Crohn's disease presenting aspalatal ulcer. Indian J Pediatr 2006;73:229-31.

10. Mahadevan $U$, Sandborn WJ. Infliximab for the treatment of orofacial Crohn's disease. Inflamm Bowel Dis 2001;7:38-42.

11. Travis SP, Stange EF, Lemann M, et al. European evidencebasedd Consensus on the management of ulserative colitis; Current management. J Crohns Colitis 2008;2:24-62. 